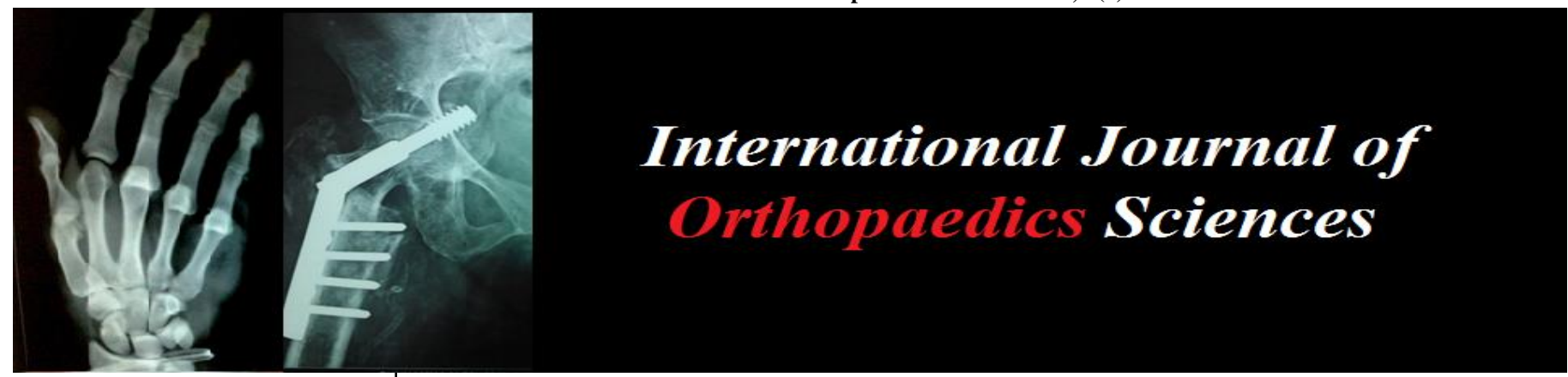

ISSN: $2395-1958$

IJOS 2019; 5(1): 87-89

(C) 2019 IJOS

www.orthopaper.com

Received: 26-11-2018

Accepted: 30-12-2018

Rajesh Kumar Ranjan

Senior resident, Department of

Orthopaedics, IGIMS, Patna,

Sheikhpura, Patna, Bihar, India

Manish Kumar

Associate prof, Department of

Orthopaedics, IGIMS, Patna,

Sheikhpura, Patna, Bihar, India

\section{Saurabh Kumar}

Senior resident, Department of

Orthopaedics, IGIMS, Patna,

Sheikhpura, Patna, Bihar, India

Santosh Kumar

Professor and Head of the

Department, Department of

Orthopaedics, IGIMS, Patna

Sheikhpura, Patna, Bihar, India

Abhijeet Subhash

Assistant prof, Department of

Orthopaedics, IGIMS, Patna,

Sheikhpura, Patna, Bihar, India

Correspondence

Rajesh Kumar Ranjan

Senior resident, Department of

Orthopaedics, IGIMS, Patna,

Sheikhpura, Patna, Bihar, India

\section{Flexor tendon injury of hand and its reconstruction}

\section{Rajesh Kumar Ranjan, Manish Kumar, Saurabh Kumar, Santosh Kumar and Abhijeet Subhash}

DOI: https://doi.org/10.22271/ortho.2019.v5.i1b.20

\section{Abstract}

The flexor system of the fingers consisting of flexor tendons and finger pulleys are a key anatomic structure for the grasping function. Athletes and manual workers are particularly at risk for closed injuries of the flexor system: ruptured pulleys, ruptures of the flexor digitorum profundus from its distal attachment ("jersey finger'), and less frequently, ruptures of the flexor digitorum superficialis and of the lumbrical muscles. In the finger, the tendons pass through tunnels that keep them close to the bones, which helps them work better. Sonography is the best imaging modality associate with the clinical exam for it allows an experienced physician to make an accurate and early diagnosis, crucial to appropriate early treatment planning. The advances in suture techniques, better understanding of the tendon morphology and its biomechanics have resulted in better outcomes.

Keywords: hand injuries, tendon repair, tendon injuries, rehabilitation

\section{Introduction}

Tendons connect muscle to bone to allow the force to be transmitted from the muscle to the bone, allowing the joint to move. The hand is divided into 5 zones Zone I starts at the end of the fingers with zone $\mathrm{V}$ at the wrist joint. Zone 1 is distal to the flexor digitorum superficialis insertion. Zone II (no mans land) between the limits of the flexor tendon sheath which is at the A1 pulley and the insertion of the flexor digitorum superficialis, which is in close proximity to the flexor tendon sheath. Zone III is from the distal edge of the transverse carpal ligament and the A1 pulley, with nerves, tendons and vessels being frequently involved due to the close proximity. Zone IV refers to the tendons within the carpal tunnel and zone $\mathrm{V}$ encompasses the forearm proximal to the carpal tunnel.

All digital flexors have origin from the medial epicondyle, anterior of radius, ulna and interosseous membrane. These tendons become tendentious in distal third of forearm and pass through the osteofibrous tunnel named carpal tunnel. Tendons of flexor digitorum superficialis (FDS) and profundus (FDP) (with flexor pollicis longus) are located deeper respectively. Tendons of FDS and FDP are enclosed in a common synovial sheath, When after entering the palm of hand, the flexor tendons fans out to their respective digits. Tendons of flexor digitorum superficialis are superficial to the flexor digitorum profundus. Hand injuries have become one of the commonest reasons for patients to visit emergency departments of hospitals. Sharp injuries leading to flexor tendon tears have always remained a challenge for doctors to bring the patients back to normal condition and saving them from prolonged disability. A torn or cut tendon in the forearm, at the wrist, in the palm, or along the finger will make it impossible to bend one or more joints in a finger. Because flexor tendons are very close to the surface of the skin, a deep cut will most likely hit a flexor tendon. In these cases, the tendon is often cut into two pieces. Because the nerves to the fingers are also very close to the tendons, a cut may damage them, as well. This will result in numbness on one or both sides of the finger. If blood vessels are also cut, the finger may have no blood supply. This requires immediate surgery. Before repairing the tendon it is essential to ascertain other possible injuries which include fracture of the phalanx and metacarpal, as well as the neurovascular damage to the involved digit. After the repair of flexor tendons achieving good range of motion is challenge while avoiding disruption of repair. Early mobilization after repair is known to prevent contractures. 


\section{Materials and Methods}

The prospective study consists of 30 patients who had undergone flexor tendon reconstruction at the department of Orthopedic surgery, IGIMS, Patna. All patients had suffered injuries of flexor tendons with considerable scarring and nonfunctioning flexor apparatus.

\section{Method of collection of data}

Relevant history followed by patient or patient's attender consent for reconstruction of flexor tendon was taken.

\section{Inclusion criteria}

- Willingness to participate and follow up.

- Clinical evaluation of flexor tendon injury by surgeon.

- Age 15-50 yr.

\section{Exclusion criteria}

- Patients with dirty or infected wounds, or those having multiple injuries other than tendons, Injury to extensor tendons were excluded from the study.

- Age less than $15 \mathrm{yr}$ and more than $50 \mathrm{yr}$.

\section{Surgical procedure}

Operation were performed under brachial block or general anesthesia with the use of a tourniquet. Perioperative antibiotic prophylaxis were given to all patients. The Bruner's zigzag incision was applied. During surgery thick flaps were raised and the tissue handling is done meticulously to prevent adhesion formation. To find the retracted tendon we tried to find the cut end of tendons in distal and proximal canals. During exploration to find tendons, we had to extend the incision. The proximal cut ends of the tendons may be retracted considerably into the palm and can be brought into the wound by milking the palm from proximal to distal end. The distal ends of the tendons can be brought into the vision by passively flexing the DIP joint. If direct suturing of the tendon is not possible then a part of tendon can be taken as a graft. Pullies were reconstructed as needed. The suture material used were nonabsorbable, $3 / 0$ or $4 / 0$ polypropylene. The tension is adjusted so that when the wrist is in extension, the finger will automatically be brought in to about the same amount or slightly more flexion in relation to the adjoining digits, flexion is to be increased a little in the more ulnar digits. After completing the flexor tendon repair, a dorsal splint is applied to keep the wrist in neutral position and metacarpophalangeal (MCP) joints in $50-70^{\circ}$ of flexion. The proximal interphalangeal (PIP) and distal interphalangeal (DIP) joints should be able to extend fully. One week after surgery, patients are started on a controlled mobilization program (passive flexion, active extension). Active ROM exercise is started at 3 weeks and unprotected digital motion is allowed at 6 weeks.

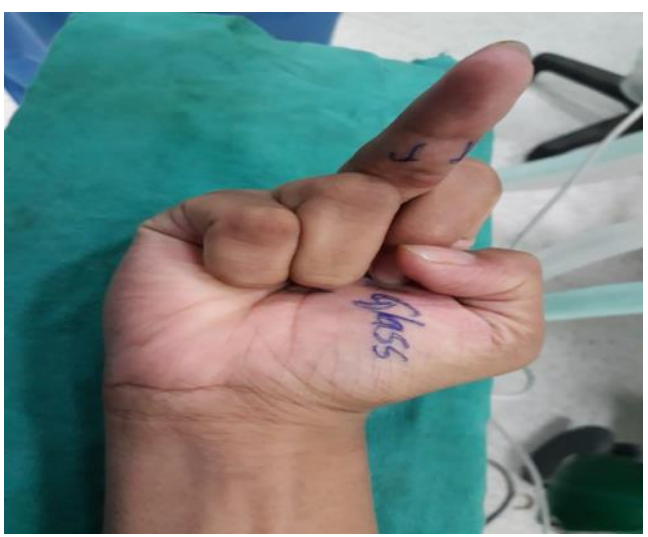

Preoperative Clinical Photograph

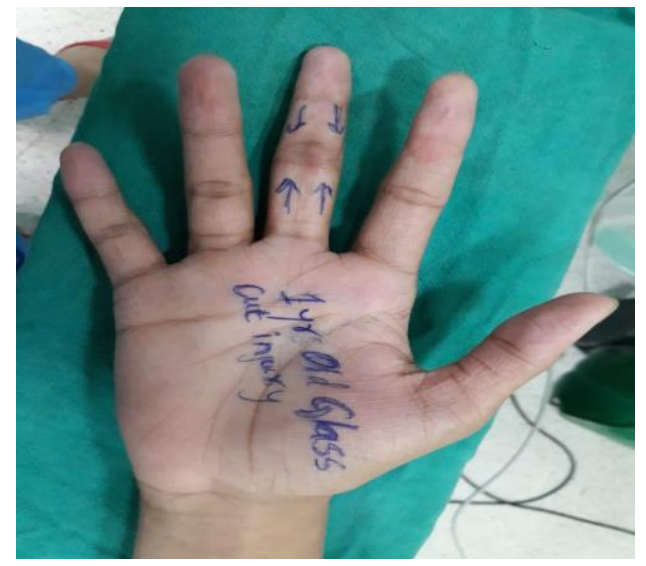

Unable To Flex Finger

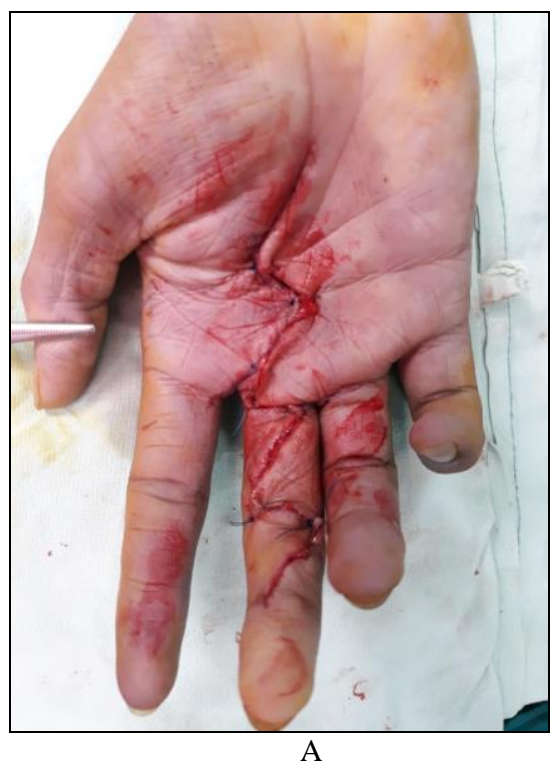

A

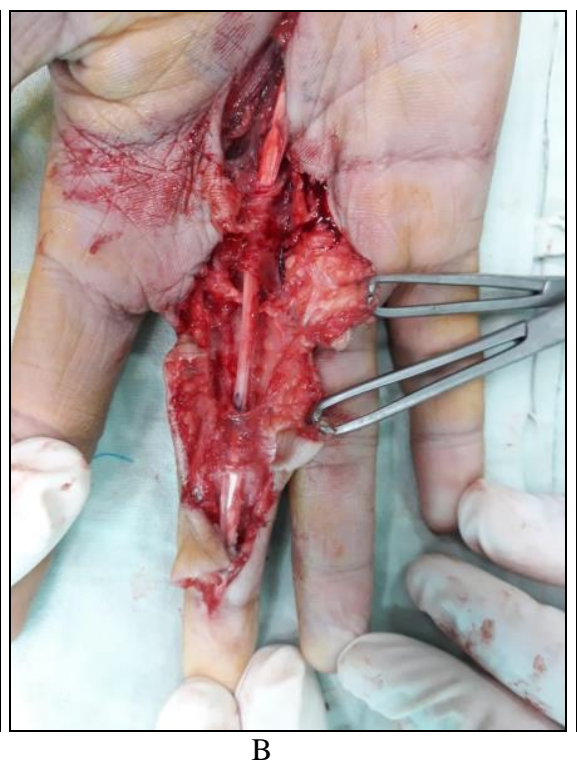

Intraoperative Photograph

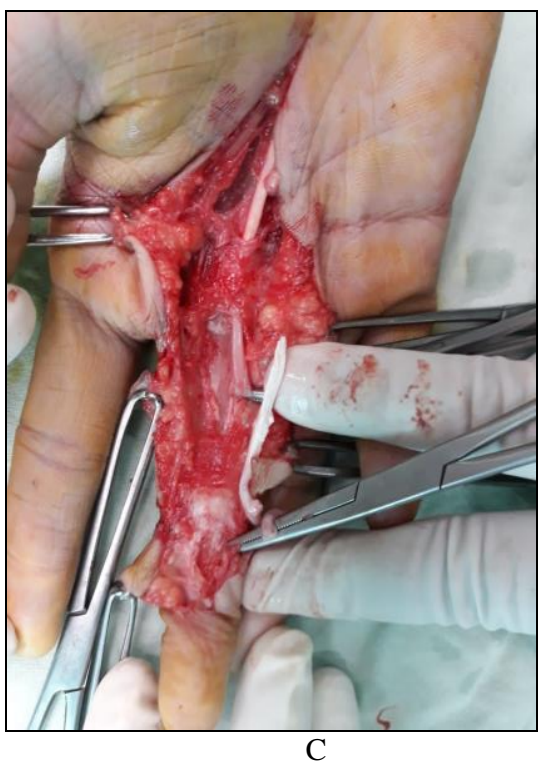

C

Discussion

Each specific movement of the hand relies on the finely tuned 
biomechanical interplay of the intrinsic and extrinsic musculotendinous forces. Restoring digital function after a flexor tendon injury continues to be one of the greatest challenges in the field of hand surgery. Flexor tendon repairs have improved with advances in the understanding of flexor tendon anatomy, biomechanics, nutrition, and healing. The optimal time for repair of the flexor tendons is within 24 hours of the injury. Bruner's zigzag incision provides excellent surgical exposure but there may be scar formation directly over the tendon. It is essential that thick flaps are raised and the tissue handling is very meticulous to prevent adhesion formation. Brunner or lateral (Midaxial) incisions are used so that adequate exposure of the flexor fibro-osseous canal can be obtained. An atraumatic technique for tendon manipulation prevents further injury to the tendons and decreases the amount of adhesion formation. Every traumatic site along the tendon is another potential spot for adhesion formation. Delicate forceps, such as the Bishop-Harmon or Iris forceps, should be used to pick up the tendon at its severed end, though not along the sides of the tendon. The goal of the tendon repair is to coapt the severed ends without bunching or leaving a gap. Increasing the repair strength through increasing the number of strands crossing the repair site to allow active postoperative mobilization without increasing the risk of rupture is logical, it can compromise tendon gliding function Furthermore, increased numbers of strands increase the tendon bulk and surface irregularity which has mechanical implications on gliding function. After flexor tendon repair there are still several complications that can occur with tendon surgery including adhesion formation, tendon rupture and stiffness of the joints. The immediate complications can include infection, tendon rupture, pulley rupture and poor tendon gliding. Rupture can be due to overload of the tendons, edema, misuse of the hand or bulky tendons. Rupture is usually seen if there is a gap, as this weakens the tendon, and increases the length. Active range of motion has shown to prevent adhesion but they may be associated with an increased rate of rupture. Later complications are also seen including tendon adhesions, decreased flexion strength, complex regional pain syndrome and abnormal scars.

\section{Conclusion}

Open and blunt tendon injuries of the hand, especially injuries of flexor tendons, are serious injuries that should be treated by a skilled surgeon. If in doubt, ultrasound and MRI can be of use for diagnosis. Initial therapy is important for a good result outcome. Close cooperation of the surgeon and the patient is essential, as well as physiotherapy and occupational therapy.

\section{References}

1. Idler RS. Anatomy and biomechanics of the digital flexor tendons. Hand Clin. 1985; 1(1):3-11. [Medline].

2. Nimbarte AD, Kaz R, Li ZM. Finger joint motion generated by individual extrinsic muscles: A cadaveric study. J Orthop Surg. 2008; 11(3):27. [Medline].

3. Moutet F, Forli A, Voulliaume D. Pulley rupture and recon-struction in rock climbers. Tech Hand Up Extrem Surg. 2004; 8:149-55.

4. Kleinert HE, Lubahn JD. Current state of flexor tendon surgery. Ann Chir Main. 1984; 3(1):7-17. [Medline].

5. Arora R, Fritz D, Zimmermann R, et al. Reconstruction of the digital flexor pulley system: a retrospective comparison of two methods of treatment. J Hand Surg [Br]. 2007; 32(1):60-6. [Medline].
6. Gelberman RH, Vande Berg JS, Lundborg GN, Akeson WH. Flexor tendon healing and restoration of the gliding surface. J Bone Joint Surg Am. 1983; 65A:70-80.

7. Strickland JW. Development of flexor tendon surgery: twenty-five years of progress. J Hand Surg [Am]. 2001; 25A:214-235.

8. Gelberman RH, Manske PR, Akeson WH, Woo SL-Y, Lundborg G, Amiel D. Flexor tendon repair. J Orthop Res. 1986; 4:119-128.

9. Mason ML, Shearon CG. Process of tendon repair: an experimental study of tendon suture and tendon graft. Arch Surg. 1932; 25:616-691.

10. Ketchum LD. Suture materials and suture techniques used in tendon repair. Symposium on flexor tendon surgery. Hand Clin. 1985; 1:43-53.

11. Dy, Christopher J, Daluiski, Aaron. Update on Zone II Flexor Tendon Injuries JAAOS - Journal of the American Academy of Orthopaedic Surgeons. 2014; 22(12):791-79

12. Evans RB. A study of the zone I flexor tendon injury and its implications for treatment. J Hand Ther. 1990; 3:133148.

13. Manske PR, Lesker PA. Avulsion of the ring finger flexor digitorum profundus tendon: an experimental study. Hand. 1978; 10:52-55.

14. McClinton MA, Curtis RM, Wilgis EF. One hundred tendon grafts for isolated flexor digitorum profundus injuries. J Hand Surg. 1982; 7A:224-229. 